\title{
LHCb full-detector real-time alignment and calibration: latest developments and perspectives
}

\author{
Agnieszka Dziurda ${ }^{1}$, Andrea Mogini ${ }^{2 * \dagger}$ \\ ${ }^{1}$ Institute of Nuclear Physics PAS, Krakow \\ ${ }^{2}$ Laboratoire de Physique Nucleaire et des Hautes Energies, Paris \\ E-mail: agnieszka.dziurda@cern.ch
}

The LHCb detector is a single-arm forward spectrometer, which measures proton-proton interactions at the LHC. For Run II (2015-2018) LHCb has introduced a novel real-time detector alignment and calibration strategy. Data collected at the start of the fill are processed and used to update the alignment, while the calibration constants are evaluated for each run. In this document an overview of the real-time alignment and calibration strategy is presented, followed by the discussion of the latest developments.

Sixth Annual Conference on Large Hadron Collider Physics (LHCP2018)

4-9 June 2018

Bologna, Italy

* Speaker.

${ }^{\dagger}$ on behalf of the LHCb collaboration 


\section{The LHCb detector and strategy of data taking}

The $\mathrm{LHCb}$ detector is a general purpose single-arm spectrometer covering the forward pseudorapidity region of $2<\eta<5[1,2]$. The detector includes a high-precision tracking system consisting of a silicon-strip vertex detector (VELO) surrounding the $p p$ interaction region, a largearea silicon-strip detector (TT) located upstream of a dipole magnet with a bending power of about $4 \mathrm{~T} \cdot \mathrm{m}$, and three stations of silicon-strip detectors (IT) and straw drift tubes (OT) placed downstream of the magnet commonly known together as $\mathrm{T}$ stations. The particle identification system is composed of two ring-imaging Cherenkov detectors (RICH1, RICH2), electromagnetic (ECAL) and hadronic (HCAL) calorimeters, and a system of muon chambers (MUON). A schematic view of the LHCb detector is shown in the left part of Fig.1 highlighting the sub-detectors which require to be either aligned or calibrated.
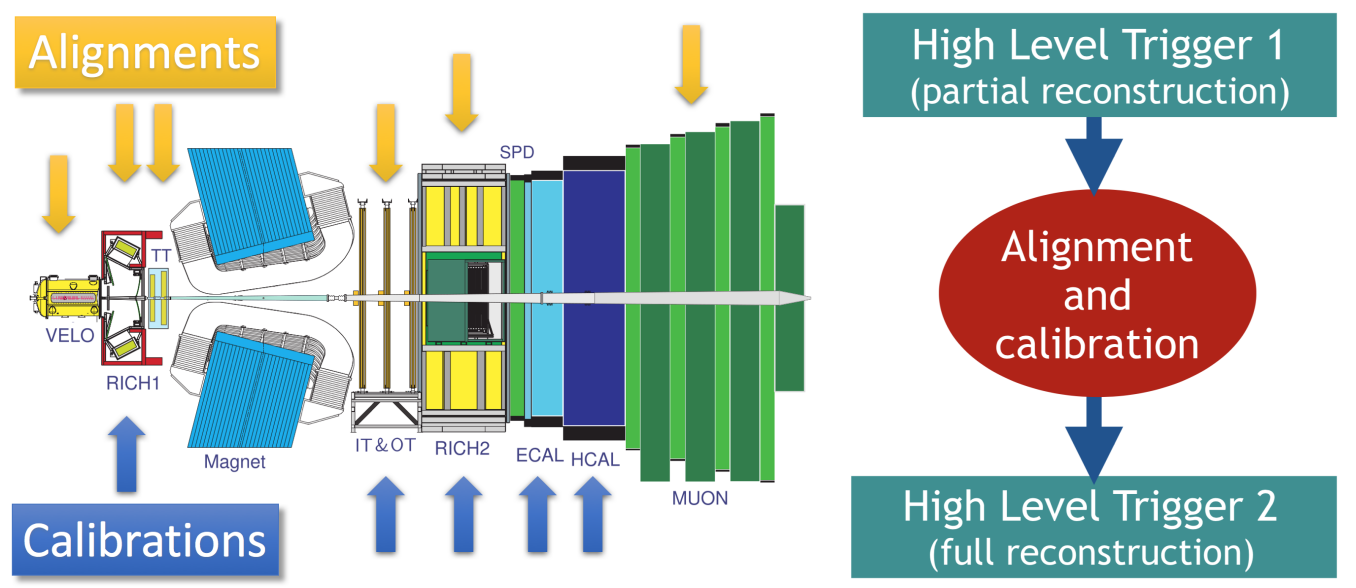

Figure 1: Left: A schematic view of the $\mathrm{LHCb}$ detector as described in the text. A blue (orange) arrows indicate the part of the detector which requires calibration (alignment). Right: the strategy of the high-level software trigger.

During Run II (2015-2018) of data taking the LHCb experiment collects data with the average bunch crossing rate of about $30 \mathrm{MHz}$. The information from the calorimeter system and muon chambers is used in the hardware trigger, which reduces the rate of accepted candidates to about $1 \mathrm{MHz}$. At this rate the detector can be read out and the passed candidates are processed further by the high-level software trigger. The simplified sketch of the high-level software trigger strategy is shown in the right part of Fig.1. The first stage (HLT1) provides the partial reconstruction of high momentum candidates. The selected events are buffered on the Event Filter Farm (EFF) and serve as an input to the alignment and calibrations tasks. Once detector is well-aligned and calibrated the second stage (HLT2) is executed where the full, offline-quality event reconstruction is performed. LHCb Run II strategy allows the selection of physics analyses to be obtained directly in the trigger [3].

\section{Real-time alignment and calibration}

A precise detector alignment and calibration plays a crucial role in obtaining the best physics performance in the HLT2. The alignment provides the best known position and orientation of the 


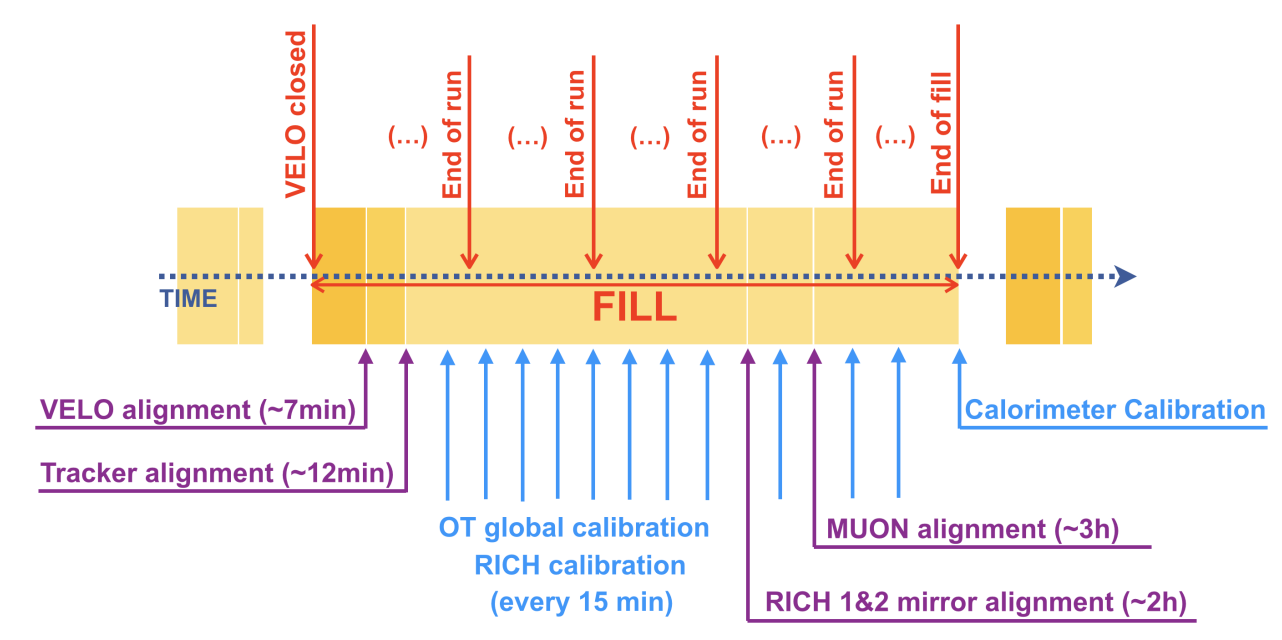

(( 7 $\mathrm{min}),(\sim 12 \mathrm{~min}),(\sim 3 \mathrm{~h}),(\sim 2 \mathrm{~h}))$ - time needed for both data accumulation and running the task

Figure 2: A schematic view of the real-time alignment and calibration procedure starting at the beginning of each fill, as for 2018 data taking.

detector elements, whereas the calibration applies necessary corrections to the detector element response. To not impact physics measurements, their accuracy must be significantly better than the resolution. The major detector alignment and calibration tasks running in the $\mathrm{LHCb}$ experiment consist of:

- the VELO alignment, followed by the alignment of the tracking stations (TT, IT, OT);

- the MUON alignment;

- the alignment of primary and secondary mirrors' rotation around local axes in both RICHes;

- global time alignment offset between the collision time and the LHCb clock that affects the drift time measured in the straw tubes which compose the OT;

- RICH gas refractive-index and Hybrid Photon Detectors calibrations;

- ECAL LED (relative) and $\pi^{0}$ (absolute) calibrations;

The precise alignment of the VELO is crucial for tracking related resolutions such as: impact parameter, primary vertex and decay-time. A more precise alignment of the entire tracking system directly implies a better invariant mass resolution. An accurate calibration and alignment of RICH detectors, improves the particle identification and allows to increase the purity of exclusive hadron selections. The calibration of the ECAL improves the particle identification and invariant mass resolution of neutral particles. Since the information from the muon system is used in the hardware trigger, an alignment precision better than $1 \mathrm{~mm}$ is required.

Alignment and calibration quantities may vary with time due to different reasons (for example pressure and temperature changes, switches of the magnet polarity, automatic or manual movements of the detector) therefore these quantities need to be evaluated as often as required. The computing power available in the Run II EFF allows to fulfill this condition. The schematic view 
of the alignment and calibration procedure of the LHCb detector is shown Fig. 2. Each automatic alignment procedure runs at the start of each fill, as soon as the required sample of candidates has been selected, saved in the buffer and reconstructed within the EFF. The tracking system uses the procedure described in Ref. [4, 5]. Since the VELO halves are closed for every fill its alignment is performed first and other sub-detectors are aligned with respect to it. Figure 3 shows the stability of the tracking system alignment for the $\mathrm{x}$ and $\mathrm{y}$ (VELO), $\mathrm{z}$ (Tracker) and $\mathrm{x}$ (MUON) translations during 2017 data taking. The horizontal dashed lines indicate the threshold within which the alignment is not updated. Each point denotes the difference between new constants and parameters from the previous alignment. As expected, the constants are updated every few fills and an overall good stability is found.

In 2017 the automatic RICH alignment procedure has been introduced, which is performed for both RICH detectors and results with 110 mirror pairs to be aligned. The alignment consists of primary and secondary mirrors' rotation around local axes, and is obtained from fitting the variation of Cherenkov angle as the function of polar angles. The stability of the RICH alignment is shown in Fig. 4 for RICH1 primary mirrors, and RICH2 secondary mirrors. An overall good stability during the year is found.
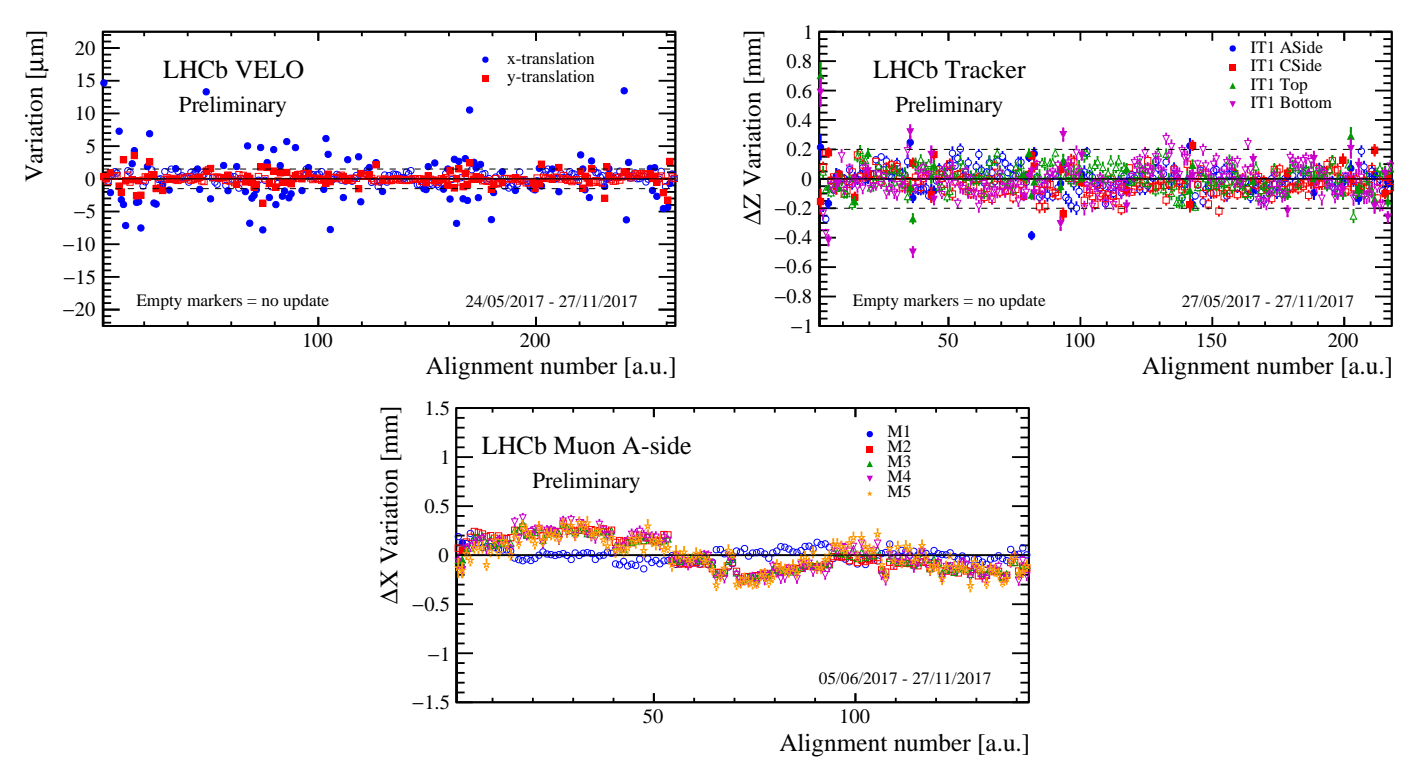

Figure 3: The stability of the VELO half (top left), Tracker (top right) and MUON (bottom) alignment during the 2017 data taking. Each point indicates the difference between the alignment constants with respect to the value from the previous alignment.

The reconstruction of electromagnetic particles such as photons, electrons, and $\pi^{0}$ mesons is performed by the calorimeters. As indicated in Fig. 2 the automatic LED calibration is performed for fills longer than 3.5 hours, where LED amplitudes are compared with the reference and, if required, High Voltage update is applied. In addition, the absolute $\pi^{0}$ calibration is processed once per month when sufficient data (300M events) is collected. Since 2018 both steps of calibrations are fully automated, allowing the same constants to be used in the trigger as in the offline analysis. The performance of the calorimeter reconstruction is compared using the example of the $B^{0} \rightarrow$ 


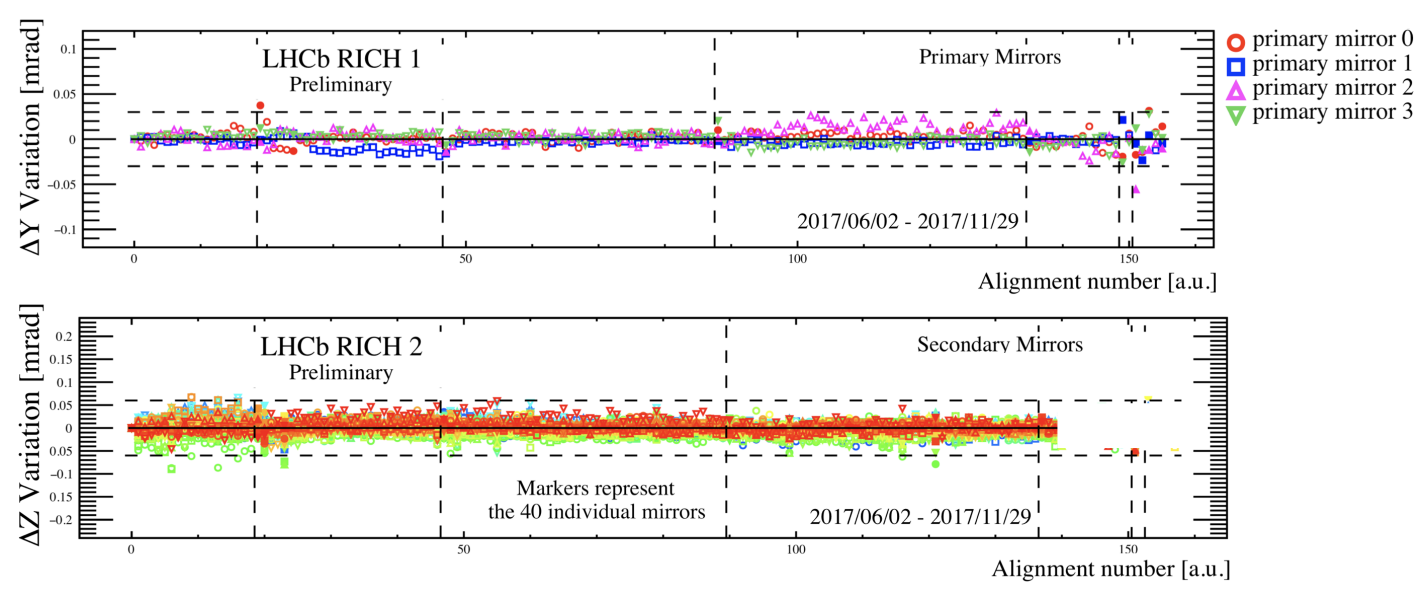

Figure 4: The stability of the RICH1 primary mirrors (top) and RICH2 secondary mirrors (bottom) alignment during the 2017 data taking. Each point indicates the difference between the alignment constants with respect to the value from the previous alignment.

$\left(K^{+} \pi^{-}\right) \gamma$ decay. The invariant mass resolution of this decay is improved by about $5 \%$ between Run I and Run II.

\section{Conclusion}

In Run II of data taking the $\mathrm{LHCb}$ detector has introduced the real-time alignment and calibration. The alignment of tracking system runs in the automated mode since 2015. In followed years other alignment tasks, as for example RICH mirrors' alignment, has been automated. In addition, since 2018 both relative and absolute calorimeter calibrations run in the automatic mode improving mass resolution of the neutral particles. The real-time alignment and calibration of the LHCb detector provides the best quality constants in HLT2, resulting with an excellent detector and physics performance achieved in already in the trigger.

\section{Acknowledgments}

Agnieszka Dziurda would like to express gratitude to the National Science Centre NCN in Poland, for financial support under the contract no. 2015/18/M/ST2/00123. Andrea Mogini acknowledges funding from the Institute Lagrange de Paris.

\section{References}

[1] Jr. Alves et al. (LHCb collaboration), JINST 3, S08005 (2008).

[2] R. Aaij et al. (LHCb collaboration), Int. J. Mod. Phys. A30, 07 1530022, (2015).

[3] A. Pearce (LHCb collaboration), PoS(LHCP2018)226, these proceedings.

[4] J. Amoraal J. et al., Nucl. Instrum. Meth. A712, $48-55$ (2013).

[5] W. Hulsbergen, Nucl. Instrum. Meth., A600, 471-477 (2009). 